\title{
A solution for the non-cooperative equilibrium problem of two person via fixed point theory
}

\author{
Tran Duc Thanh', Aatef Hobiny ${ }^{2}$ and Erdal Karapınar ${ }^{3,4^{*}}$
}

\begin{tabular}{l}
\hline${ }^{*}$ Correspondence: \\
erdalkarapinar@yahoo.com \\
${ }^{3}$ Department of Mathematics, \\
Atilim University, Incek, Ankara, \\
06836, Turkey \\
${ }^{4}$ Nonlinear Analysis and Applied \\
Mathematics Research Group \\
(NAAM), King Abdulaziz University, \\
Jeddah, 21589, Saudi Arabia \\
Full list of author information is \\
available at the end of the article
\end{tabular}

available at the end of the article

\begin{abstract}
In this paper, we investigate the non-cooperative equilibrium problem of two person games in the setting of game theory and propose a solution via coupled fixed point results in the context of partial metric spaces. We also realize that our coupled fixed point results can be applied to get a solution of a class of nonlinear Fredholm type integral equations.
\end{abstract}

MSC: 46J10;46J15; 47H10

Keywords: coupled fixed point; partial metric; F-contractions; non-cooperative equilibrium

\section{Introduction}

It is very well known fact that real world problem can be modeled as a mathematical equation. The existence of a solution of such problems has been investigated in several branches of mathematics, such as differential equations, integral equations, functional equations, partial differential equations, random differential equations, etc. and one has proposed solutions for such problems via fixed point theory. But the application area of fixed point theory is not only limited to mathematics, but also occurs in other quantitative sciences, such as, computer science, economics, biology, physics, etc. Game theory, a branch of economics, has used fixed point theory techniques and approaches to solve its own problems.

Game theory can be regarded as a formal (mathematical) way to study games. Indeed, we consider the games as conflicts where some number of individuals (called players) take part and each one tries to maximize his utility in taking part in the conflict. Games can be classified in many ways, but here we focus on the following classification: Cooperative games, in which, players are allowed to cooperate and non-cooperative games, in which players are not allowed to cooperate. In the sequel, we shall demonstrate how the question of the existence of equilibria is related to the question of the existence of a fixed point. Throughout the paper, we follow the notion and notation in [1]. We recall some basic concepts.

A two person game $\mathcal{G}$ in normal form consists of the following data:

(1) topological spaces $\mathcal{S}_{1}$ and $\mathcal{S}_{2}$, the so called strategies for player 1 resp. player 2, 
(2) a topological subspace $U \subset \mathcal{S}_{1} \times \mathcal{S}_{2}$ of an allowed strategy pair,

(3) a biloss operator

$$
\begin{aligned}
L: U \rightarrow \mathbb{R}^{2} \\
\quad\left(s_{1}, s_{2}\right) \mapsto\left(L_{1}\left(s_{1}, s_{2}\right) ; L_{2}\left(s_{1}, s_{2}\right)\right),
\end{aligned}
$$

where $L_{i}\left(s_{1}, s_{2}\right)$ is the loss of player $i$ if the strategies $s_{1}$ and $s_{2}$ are played.

A pair $\left(\bar{s}_{1}, \bar{s}_{2}\right) \in U$ is called a non-cooperative equilibrium if

$$
\begin{aligned}
& L_{1}\left(\bar{s}_{1}, \bar{s}_{2}\right) \leq L_{1}\left(s_{1}, \bar{s}_{2}\right), \quad \forall s_{1} \in S_{1}, \\
& L_{2}\left(\bar{s}_{1}, \bar{s}_{2}\right) \leq L_{2}\left(\bar{s}_{1}, s_{2}\right), \quad \forall s_{2} \in S_{2} .
\end{aligned}
$$

Assume that there exist mappings

$$
\begin{aligned}
& C: \mathcal{S}_{2} \rightarrow \mathcal{S}_{1}, \\
& D: \mathcal{S}_{1} \rightarrow \mathcal{S}_{2},
\end{aligned}
$$

such that the following equations hold:

$$
\begin{array}{ll}
L_{1}\left(C\left(s_{2}\right), s_{2}\right)=\min _{s_{1} \in S_{1}} L_{1}\left(s_{1}, s_{2}\right), & \forall s_{2} \in S_{2}, \\
L_{2}\left(s_{1}, D\left(s_{1}\right)\right)=\min _{s_{2} \in S_{2}} L_{2}\left(s_{1}, s_{2}\right), & \forall s_{1} \in S_{1} .
\end{array}
$$

Such mappings $C$ and $D$ are called optimal decision rules. Then any solution $\left(\bar{s}_{1}, s_{2}\right)$ of the system

$$
\begin{aligned}
& C\left(\bar{s}_{2}\right)=\bar{s}_{1}, \\
& D\left(\bar{s}_{1}\right)=\bar{s}_{2},
\end{aligned}
$$

is a non-cooperative equilibrium. Denoting by $F$ the function

$$
\begin{aligned}
F: \mathcal{S}_{1} \times \mathcal{S}_{2} & \rightarrow \mathcal{S}_{1} \times \mathcal{S}_{2} \\
\left(\bar{s}_{1}, \bar{s}_{2}\right) & \mapsto\left(C\left(\bar{s}_{2}\right) ; D\left(\bar{s}_{1}\right)\right),
\end{aligned}
$$

any coupled fixed point $\left(\bar{s}_{1}, \bar{s}_{2}\right)$ of $F$ is a non-cooperative equilibrium. Hence, the investigation of the existence of a solution for a non-cooperative equilibrium is equivalent to searching for the existence of a couple fixed point. More details as regards game theory can be found in [1].

The main goal of the present work is to solve the problem of the non-cooperative equilibrium of two person games. For this purpose, we shall present some coupled fixed point theorems in partial metric spaces. Our aim is to explore not only the results themselves but also their applications to nonlinear integral equations.

\section{Preliminaries}

The notion of a partial metric was proposed by Matthews (see [2, 3]) as a generalization of the metric concept to get better results in the branches of computer sciences: semantics 
and computer domain. Indeed, a partial metric is a function that is obtained from the metric by replacing the condition $d(x, x)=0$ with the condition $d(x, x) \leq d(x, y)$ for all $x, y$. In the last decade, a number of authors have brought into focus fixed point problems in the context of partial metric spaces as well as topological properties of a partial metric space; see e.g. [4-14] and the related references given therein.

We first need to recall some basic concepts and necessary results. Throughout the paper, $\mathbb{N}$ and $\mathbb{N}_{0}$ denote the set of positive integers and the set of nonnegative integers, respectively. Similarly, $\mathbb{R}, \mathbb{R}^{+}$, and $\mathbb{R}_{0}^{+}$represent the set of reals, positive reals, and nonnegative reals, respectively.

Definition 2.1 (See e.g. [2,5]) Let $X$ be a nonempty set. The mapping $p: X \times X \rightarrow[0, \infty)$ is said to be a partial metric on $X$ if for any $x, y, z \in X$ the following conditions hold true:

(P1) $x=y$ if and only if $p(x, x)=p(y, y)=p(x, y)$.

(P2) $p(x, x) \leq p(x, y)$.

(P3) $p(x, y)=p(y, x)$.

(P4) $p(x, z) \leq p(x, y)+p(y, z)-p(y, y)$.

The pair $(X, p)$ is then called a partial metric space (in short, PMS).

Let $(X, p)$ be a partial metric space. Then the functions $d_{p}, d_{m}: X \times X \rightarrow[0, \infty)$ given by

$$
d_{p}(x, y)=2 p(x, y)-p(x, x)-p(y, y)
$$

and

$$
d_{m}(x, y)=\max \{p(x, y)-p(x, x), p(x, y)-p(y, y)\}
$$

are the (usual) metrics on $X$. It is easy to check that $d_{p}$ and $d_{m}$ are equivalent. Note that each partial metric $p$ on $X$ generates a $T_{0}$-topology $\tau_{p}$ with as a base the family of open $p$-balls $\left\{B_{p}(x, \varepsilon): x \in X, \varepsilon>0\right\}$, where $B_{p}(x, \varepsilon)=\{y \in X: p(x, y)<p(x, x)+\varepsilon\}$.

Definition 2.2 (See e.g. $[4,5])$ Let $(X, p)$ be a partial metric space.

(1) A sequence $\left\{x_{n}\right\}$ in $X$ converges to $x \in X$ if and only if $p(x, x)=\lim _{n \rightarrow \infty} p\left(x_{n}, x\right)$.

(2) A sequence $\left\{x_{n}\right\}$ in $X$ is called a Cauchy sequence if and only if $\lim _{n, m \rightarrow \infty} p\left(x_{n}, x_{m}\right)$ exists (and is finite).

(3) $(X, p)$ is called complete if every Cauchy sequence $\left\{x_{n}\right\}$ in $X$ converges to $x \in X$.

(4) A mapping $f: X \rightarrow X$ is said to be continuous at $x_{0} \in X$ if, for every $\varepsilon>0$, there exists $\delta>0$ such that $f\left(B\left(x_{0}, \delta\right)\right) \subset B\left(f\left(x_{0}\right), \varepsilon\right)$.

Referring to [15], we say that a sequence $\left\{x_{n}\right\}$ in $(X, p)$ is called a 0 -Cauchy sequence if $\lim _{n, m \rightarrow \infty} p\left(x_{n}, x_{m}\right)=0$. Also, we say that $(X, p)$ is 0 -complete if every 0 -Cauchy sequence in $X$ converges, with respect to the partial metric $p$, to a point $x \in X$ such that $p(x, x)=0$. Notice that if $(X, p)$ is complete, then it is 0 -complete, but the converse does not hold. Moreover, every 0 -Cauchy sequence in $(X, p)$ is Cauchy in $\left(X, d_{p}\right)$.

Example 2.3 (See e.g. [2,15])

(1) Let $X=[0,+\infty)$ and define $p(x, y)=\max \{x, y\}$, for all $x, y \in X$. Then $(X, p)$ is a complete partial metric space. It is clear that $p$ is not a (usual) metric. 
(2) Let $X=[0,+\infty) \cap \mathbb{Q}$, where $\mathbb{Q}$ is the set of rational numbers. Define $p(x, y)=\max \{x, y\}$, for all $x, y \in X$. Then $(X, p)$ is a 0 -complete partial metric space which is not complete.

Proposition 2.4 (See e.g. $[4,5])$ Let $(X, p)$ be a partial metric space.

(1) A sequence $\left\{x_{n}\right\}$ is a Cauchy sequence in $(X, p)$ if and only if $\left\{x_{n}\right\}$ is a Cauchy sequence in $\left(X, d_{p}\right)$.

(2) $(X, p)$ is complete if and only if $\left(X, d_{p}\right)$ complete. Moreover,

$$
\lim _{n \rightarrow \infty} d_{p}\left(x_{n}, x\right)=0 \Leftrightarrow \lim _{n \rightarrow \infty} p(x, x)=\lim _{n \rightarrow \infty} p\left(x_{n}, x\right)=\lim _{n, m \rightarrow \infty} p\left(x_{m}, x_{n}\right) .
$$

The following lemmas have an important role to play in the proofs of the theorems.

Lemma 2.5 (See e.g. $[4,8]$ ) Assume $x_{n} \rightarrow z$ as $n \rightarrow \infty$ in a PMS $(X, p)$ such that $p(z, z)=0$. Then $\lim _{n \rightarrow \infty} p\left(x_{n}, y\right)=p(z, y)$ for every $y \in X$.

Lemma 2.6 (See e.g. $[4,8])$ Let $(X, p)$ be a complete PMS. Then:

(1) If $p(x, y)=0$ then $x=y$.

(2) If $x \neq y$, then $p(x, y)>0$.

Lemma 2.7 (See e.g. $[4,8])$ Let $(X, p)$ be a PMS. If $x_{n} \rightarrow x$ and $y_{n} \rightarrow y$ as $n \rightarrow \infty$ for all $x_{n}, y_{n}, x, y \in X$ then $p\left(x_{n}, y_{n}\right) \rightarrow p(x, y)$ as $n \rightarrow \infty$.

The existence and uniqueness of fixed points of contractive type mappings in partially ordered metric spaces have been considered recently by several authors: Ran and Reurings [16], Nieto and Rodriguez-Lopez $[17,18]$. On the other hand, the notion of a coupled fixed point was suggested by Guo and Lakshmikantham in [19]. Following this initial result, Gnana Bhaskar and Lakshmikantham [20] proposed the notion of mixed monotone property and get coupled fixed point results in the setting of partially ordered metric spaces (see also [21-23] and the related references therein.) Later, it was reported that most of the coupled fixed point results can be derived from the existence results, and vice versa; see e.g. [24-26]. On the other hand, coupled fixed point results still have worth regarding their applications. Most of the times, using coupled fixed point theory is the most economical way to solve problems (regarding time and speed of the process). This paper can be considered as an example.

Recall that a pair $(x, y) \in X \times X$ is called a coupled fixed point of the mapping $T: X \times X \rightarrow$ $X$ if $T(x, y)=x, T(y, x)=y$ (see e.g. [19]).

Definition 2.8 ([20]) Let $(X, \leq)$ be a partially ordered set and $T: X \times X \rightarrow X$. The mapping $T$ is said to have the mixed monotone property if $T(x, y)$ is monotone non-decreasing in $x$ and monotone non-increasing in $y$, that is, for any $x, y \in X$

$$
x_{1}, x_{2} \in X, \quad x_{1} \leq x_{2} \quad \Rightarrow \quad T\left(x_{1}, y\right) \leq T\left(x_{2}, y\right)
$$

and

$$
y_{1}, y_{2} \in X, \quad y_{1} \leq y_{2} \quad \Rightarrow \quad T\left(x, y_{1}\right) \geq T\left(x, y_{2}\right) .
$$


Next, we introduce a class of functions which plays a crucial role in this paper. Let $F$ : $\mathbb{R}_{0}^{+} \rightarrow \mathbb{R}$ be a mapping satisfying:

$\left(\mathrm{F}_{1}\right) F$ is strictly increasing and continuous.

$\left(\mathrm{F}_{2}\right)$ For each sequence $\left(a_{n}\right) \subset \mathbb{R}_{0}^{+}, \lim _{n \rightarrow \infty} a_{n}=0$ if and only if $\lim _{n \rightarrow \infty} F\left(a_{n}\right)=-\infty$.

We denote by $\mathcal{F}$ the family of all functions $F$ that satisfy the conditions $\left(\mathrm{F}_{1}\right)-\left(\mathrm{F}_{2}\right)$ (see [27]). It is easy to check that $F(x)=\ln x$ and $G(x)=\ln x+x$ for all $x \in \mathbb{R}_{0}^{+}$belong to $\mathcal{F}$.

In [28], Wardowski introduced the new concept of an $F$-contraction and proved fixed point theorems in the classical setting of metric spaces. In [27], the authors introduced the concept of an $F$-contraction, a generalized $F$-contraction, and they proved some fixed point theorems for multi-valued mappings in the partial metric spaces (see also [6, 9, 29-32]).

Definition 2.9 ([27]) Let $(X, p)$ be a partial metric space. A mapping $T: X \times X \rightarrow X$ is called an $F$-contraction if there exist $F \in \mathcal{F}$ and $\tau \in \mathbb{R}_{0}^{+}$such that

$$
\tau+F(p(T x, T y)) \leq F(p(x, y))
$$

for all $x, y \in X$.

\section{Auxiliary results: coupled fixed points in partial metric spaces}

In this section we state and prove some new coupled fixed point results for $F$-contractive mappings in the context of complete partial metric spaces.

Theorem 3.1 Let $(X, \leq)$ be a partially ordered set and suppose there exists a partial metric $p$ on $X$ such that $(X, p)$ is a 0 -complete partial metric space. Let $T: X \times X \rightarrow X$ be a continuous mapping having the mixed monotone property on $X$. Suppose also that

(1)

$$
\tau+F(p(T(x, y), T(u, v))) \leq F(\max \{p(x, u), p(y, v)\})
$$

for all $x \leq u, y \geq v$, for some $F \in \mathcal{F}$ and $\tau>0$.

(2) There are $x_{0}, y_{0} \in X$ such that $x_{0} \leq T\left(x_{0}, y_{0}\right), y_{0} \geq T\left(y_{0}, x_{0}\right)$.

Then $T$ has a coupled fixed point, that is, there exist $x, y \in X$ such that $x=T(x, y), y=$ $T(y, x)$.

Proof Let $x_{0}, y_{0} \in X$ be such that $x_{0} \leq T\left(x_{0}, y_{0}\right), y_{0} \geq T\left(y_{0}, x_{0}\right)$. Let $x_{1}=T\left(x_{0}, y_{0}\right)$ and $y_{1}=$ $T\left(y_{0}, x_{0}\right)$. Then $x_{0} \leq x_{1}$ and $y_{0} \geq y_{1}$. Again, let $x_{2}=T\left(x_{1}, y_{1}\right)$ and $y_{2}=T\left(y_{1}, x_{1}\right)$. Since $T$ has the mixed monotone property, we have $x_{1} \leq x_{2}$ and $y_{1} \geq y_{2}$. Continuing this way, we get two sequences $\left\{x_{n}\right\}$ and $\left\{y_{n}\right\}$ in $X$ such that $x_{n+1}=T\left(x_{n}, y_{n}\right), y_{n+1}=T\left(y_{n}, x_{n}\right)$ and

$$
x_{0} \leq x_{1} \leq x_{2} \leq \cdots \leq x_{n} \leq x_{n+1} \cdots, \quad y_{0} \geq y_{1} \geq y_{2} \geq \cdots \geq y_{n} \geq y_{n+1} \geq \cdots .
$$

Now, for each $n=0,1,2, \ldots$, we have

$$
\begin{aligned}
\tau+F\left(p\left(x_{n}, x_{n+1}\right)\right) & =\tau+F\left(p\left(T\left(x_{n-1}, y_{n-1}\right), T\left(x_{n}, y_{n}\right)\right)\right) \\
& \leq F\left(\max \left\{\left(p\left(x_{n-1}, x_{n}\right)\right), p\left(y_{n-1}, y_{n}\right)\right\}\right)
\end{aligned}
$$


and

$$
\begin{aligned}
\tau+F\left(p\left(y_{n}, y_{n+1}\right)\right) & =\tau+F\left(p\left(T\left(y_{n-1}, x_{n-1}\right), T\left(y_{n}, x_{n}\right)\right)\right) \\
& \leq F\left(\max \left\{p\left(y_{n-1}, y_{n}\right), p\left(x_{n-1}, x_{n}\right)\right\}\right) .
\end{aligned}
$$

Since (8), (9) hold and $F$ is increasing we get

$$
\tau+F\left(\max \left\{p\left(x_{n}, x_{n+1}\right), p\left(y_{n}, y_{n+1}\right)\right\}\right) \leq F\left(\max \left\{p\left(y_{n-1}, y_{n}\right), p\left(x_{n-1}, x_{n}\right)\right\}\right) .
$$

It follows that

$$
\max \left\{p\left(x_{n}, x_{n+1}\right), p\left(y_{n}, y_{n+1}\right)\right\} \leq \max \left\{p\left(y_{n-1}, y_{n}\right), p\left(x_{n-1}, x_{n}\right)\right\}
$$

for all $n=1,2, \ldots$. Hence, the sequence $r_{n}:=\max \left\{p\left(x_{n}, x_{n+1}\right), p\left(y_{n}, y_{n+1}\right)\right\}$ is a non-increasing. Thus, there is $r \geq 0$ such that $\lim _{n \rightarrow \infty} r_{n}=r$. Since $F$ is continuous, letting $n \rightarrow \infty$ in (10), we arrive at

$$
\tau+F(r) \leq F(r) .
$$

Since $\tau>0$ and the definition of $F$, we can deduce that $F(r)=-\infty$; this implies that $r=0$. Therefore

$$
\lim _{n \rightarrow \infty} \max \left\{p\left(x_{n}, x_{n+1}\right), p\left(y_{n}, y_{n+1}\right)\right\}=0 .
$$

Next, we claim that

$$
\lim _{m, n \rightarrow \infty} \max \left\{p\left(x_{m}, x_{n}\right), p\left(y_{n}, y_{m}\right)\right\}=0 .
$$

Suppose, to the contrary, that there exists $\varepsilon>0$ for which we can seek two subsequences $\left\{x_{m(k)}\right\}$ and $\left\{x_{n(k)}\right\}$ of, respectively, $\left\{x_{m}\right\}$ and $\left\{x_{n}\right\}$ such that $n(k)$ is the smallest index for which

$$
n(k)>m(k)>k, \quad \max \left\{p\left(x_{m(k)}, x_{n(k)}\right), p\left(y_{n(k)}, y_{m(k)}\right)\right\} \geq \varepsilon .
$$

This means that

$$
\max \left\{p\left(x_{m(k)}, x_{n(k)-1}\right), p\left(y_{m(k)}, y_{n(k)-1}\right)\right\}<\varepsilon,
$$

and we obtain

$$
\begin{aligned}
p\left(x_{m(k)}, x_{n(k)}\right) & \leq p\left(x_{m(k)}, x_{n(k)-1}\right)+p\left(x_{n(k)-1}, x_{n(k)}\right)-p\left(x_{n(k)-1}, x_{n(k)-1}\right) \\
& \leq p\left(x_{m(k)}, x_{n(k)-1}\right)+p\left(x_{n(k)-1}, x_{n(k)}\right)<\varepsilon+p\left(x_{n(k)-1}, x_{n(k)}\right) .
\end{aligned}
$$

Similarly, we get

$$
p\left(y_{m(k)}, y_{n(k)}\right)<\varepsilon+p\left(y_{n(k)-1}, y_{n(k)}\right) .
$$


Combining (13), (15), and (16), we obtain

$$
\varepsilon \leq \max \left\{p\left(x_{m(k)}, x_{n(k)}\right), p\left(y_{m(k)}, y_{n(k)}\right)\right\} \leq \varepsilon+\max \left\{p\left(x_{n(k)-1}, x_{n(k)}\right), p\left(y_{n(k)-1}, y_{n(k)}\right)\right\} .
$$

Letting $k \rightarrow \infty$ in (17) and using (11), we have

$$
\lim _{k \rightarrow \infty} \max \left\{p\left(x_{m(k)}, x_{n(k)}\right), p\left(y_{m(k)}, y_{n(k)}\right)\right\}=\varepsilon
$$

Now, by the facts that

$$
p\left(x_{m(k)}, x_{n(k)}\right) \leq p\left(x_{m(k)}, x_{n(k)-1}\right)+p\left(x_{n(k)-1}, x_{n(k)}\right)
$$

and

$$
p\left(y_{m(k)}, y_{n(k)}\right) \leq p\left(y_{m(k)}, y_{n(k)-1}\right)+p\left(y_{n(k)-1}, y_{n(k)}\right)
$$

we obtain

$$
\begin{aligned}
\max \left\{p\left(x_{m(k)}, x_{n(k)}\right), p\left(y_{m(k)}, y_{n(k)}\right)\right\} \leq & \max \left\{p\left(x_{m(k)}, x_{n(k)-1}\right), p\left(y_{m(k)}, y_{n(k)-1}\right)\right\} \\
& +\max \left\{p\left(x_{n(k)-1}, x_{n(k)}\right), p\left(y_{n(k)-1}, y_{n(k)}\right)\right\} .
\end{aligned}
$$

By the same argument, we also have

$$
\begin{aligned}
\max \left\{p\left(x_{m(k)}, x_{n(k)-1}\right), p\left(y_{m(k)}, y_{n(k)-1}\right)\right\} \leq & \max \left\{p\left(x_{m(k)}, x_{n(k)}\right), p\left(y_{m(k)}, y_{n(k)}\right)\right\} \\
& +\max \left\{p\left(x_{n(k)-1}, x_{n(k)}\right), p\left(y_{n(k)-1}, y_{n(k)}\right)\right\} .
\end{aligned}
$$

Letting $k \rightarrow \infty$ in (19), (20) and using (11), (18), we have

$$
\lim _{k \rightarrow \infty} \max \left\{p\left(x_{m(k)}, x_{n(k)-1}\right), p\left(y_{m(k)}, y_{n(k)-1}\right)\right\}=\varepsilon
$$

Next, since $x_{m(k)} \leq x_{n(k)-1}$ and $y_{m(k)} \geq y_{n(k)-1}$, we have

$$
\begin{aligned}
\tau+F\left(p\left(x_{m(k)+1}, x_{n(k)}\right)\right) & =\tau+F\left(p\left(T\left(x_{m(k)}, y_{m(k)}\right), T\left(x_{n(k)-1}, x_{n(k)-1}\right)\right)\right) \\
& \leq F\left(\max \left\{p\left(x_{m(k)}, x_{n(k)-1}\right), p\left(y_{n(k)-1}, y_{m(k)}\right)\right\}\right)
\end{aligned}
$$

For the same reason, we also have

$$
\begin{aligned}
\tau+F\left(p\left(y_{n(k)}, y_{m(k)+1}\right)\right) & =\tau+F\left(p\left(T\left(y_{m(k)}, x_{m(k)}\right), T\left(y_{n(k)-1}, x_{n(k)-1}\right)\right)\right) \\
& \leq F\left(\max \left\{p\left(x_{n(k)-1}, x_{m(k)}\right), p\left(y_{n(k)-1}, y_{m(k)}\right)\right\}\right) .
\end{aligned}
$$

Therefore

$$
\begin{aligned}
\tau+ & \max \left\{F\left(p\left(x_{m(k)+1}, x_{n(k)}\right)\right), F\left(p\left(y_{n(k)}, y_{m(k)+1}\right)\right)\right\} \\
& \leq F\left(\max \left\{p\left(x_{n(k)-1}, x_{m(k)}\right), p\left(y_{n(k)-1}, y_{m(k)}\right)\right\}\right) .
\end{aligned}
$$


Letting $k \rightarrow \infty$ and using (21), we arrive at

$$
\tau+F(\varepsilon) \leq F(\varepsilon) .
$$

This yields $\varepsilon=0$, this is a contradiction. Hence, we have proved that

$$
\lim _{m, n \rightarrow \infty} \max \left\{p\left(x_{m}, x_{n}\right), p\left(y_{n}, y_{m}\right)\right\}=0 .
$$

This implies that

$$
\lim _{m, n \rightarrow \infty} p\left(x_{m}, x_{n}\right)=0 \quad \text { and } \quad \lim _{m, n \rightarrow \infty} p\left(y_{m}, y_{n}\right)=0 .
$$

Since $(X, p)$ is 0 -complete partial metric space, we can find $u, v \in X$ such that

$$
\lim _{n \rightarrow \infty} p\left(u, x_{n}\right)=p(u, u)=0
$$

and

$$
\lim _{n \rightarrow \infty} p\left(v, y_{n}\right)=p(v, v)=0
$$

Now, we show that $u=T(u, v)$ and $v=T(v, u)$. Indeed, since $u \leq u$ and $v \geq v$, we have

$$
\tau+F(p(T(u, v), T(u, v))) \leq F(\max \{p(u, u), p(v, v)\})=F(0)=-\infty .
$$

This implies that $p(T(u, v), T(u, v))=0$.

Since $x_{n} \rightarrow u, y_{n} \rightarrow v$ as $n \rightarrow \infty$ in $(X, p)$ and $T$ is continuous, we have $T\left(x_{n}, y_{n}\right) \rightarrow$ $T(u, v)$ in $(X, p)$, this means that

$$
\lim _{n \rightarrow \infty} p\left(x_{n+1}, T(u, v)\right)=\lim _{n \rightarrow \infty} p\left(T\left(x_{n}, y_{n}\right), T(u, v)\right)=0 .
$$

Now, we have

$$
p(u, T(u, v)) \leq p\left(u, x_{n+1}\right)+p\left(x_{n+1}, T(u, v)\right)-p\left(x_{n+1}, x_{n+1}\right) .
$$

Letting $n \rightarrow \infty$, we get $p(u, T(u, v))=0$, and so $u=T(u, v)$. By the same argument, we also have $p(v, T(v, u))=0$, and so $v=T(v, u)$.

In the next theorem, we omit the continuity hypothesis of $T$.

Theorem 3.2 Let $(X, \leq)$ be a partially ordered set and suppose there exists a partial metric $p$ on $X$ such that $(X, p)$ is a 0 -complete partial metric space. Let $T: X \times X \rightarrow X$ be a mapping having the mixed monotone property on $X$. Assume that:

(1)

$$
\tau+F(p(T(x, y), T(u, v))) \leq F(\max \{p(x, u), p(y, v)\})
$$

for all $x \leq u, y \geq v$, for some $F \in \mathcal{F}$ and $\tau>0$. 
(2) There are $x_{0}, y_{0} \in X$ such that $x_{0} \leq T\left(x_{0}, y_{0}\right), y_{0} \geq T\left(y_{0}, x_{0}\right)$.

Also, assume that $X$ has the properties:

(i) If a non-decreasing sequence $\left\{x_{n}\right\}$ in $X$ converges to $x$ then $x_{n} \leq x$ for all $n$.

(ii) If a non-increasing sequence $\left\{y_{n}\right\}$ in $X$ converges to $y$ then $y_{n} \geq y$ for all $n$.

Then $T$ has a coupled fixed point, that is, there exist $x, y \in X$ such that $x=T(x, y), y=$ $T(y, x)$.

Proof We follow the line of the proof of Theorem 3.1. Hence, we only need to show that

$$
\lim _{n \rightarrow \infty} p\left(x_{n+1}, F(u, v)\right)=\lim _{n \rightarrow \infty} p\left(T\left(x_{n}, y_{n}\right), T(u, v)\right)=0
$$

under conditions (i) and (ii). Indeed, we have $x_{n} \leq u$ and $y_{n} \geq v$ for all $n$. Applying (7), we have

$$
\tau+F\left(p\left(x_{n+1}, T(u, v)\right)\right)=\tau+F\left(p\left(F\left(x_{n}, y_{n}\right), F(u, v)\right)\right) \leq F\left(\max \left\{p\left(x_{n}, u\right), p\left(y_{n}, v\right)\right\}\right)
$$

Letting $n \rightarrow \infty$, we obtain

$$
\lim _{n \rightarrow \infty} F\left(p\left(x_{n+1}, T(u, v)\right)\right)=-\infty
$$

Hence

$$
\lim _{n \rightarrow \infty} p\left(x_{n+1}, F(u, v)\right)=\lim _{n \rightarrow \infty} p\left(T\left(x_{n}, y_{n}\right), T(u, v)\right)=0 .
$$

We easily get the following corollary.

Corollary 3.3 Let $(X, \leq)$ be a partially ordered set and suppose there exists a partial metric $p$ on $X$ such that $(X, p)$ is a 0-complete partial metric space. Let $T: X \times X \rightarrow X$ be a mapping having the mixed monotone property on $X$. Assume that:

(1)

$$
\tau+F(p(T(x, y), T(u, v))) \leq F\left(\frac{p(x, u)+p(y, v)}{2}\right)
$$

for all $x \leq u, y \geq v$, for some $F \in \mathcal{F}$ and $\tau>0$.

(2) There are $x_{0}, y_{0} \in X$ such that $x_{0} \leq T\left(x_{0}, y_{0}\right), y_{0} \geq T\left(y_{0}, x_{0}\right)$.

Also, assume that either

(a) $T$ is continuous; or

(b) $X$ has the properties:

(i) If a non-decreasing sequence $\left\{x_{n}\right\}$ in $X$ converges to $x$ then $x_{n} \leq x$ for all $n$.

(ii) If a non-increasing sequence $\left\{y_{n}\right\}$ in $X$ converges to $y$ then $y_{n} \geq y$ for all $n$.

Then $T$ has a coupled fixed point, that is, there exist $x, y \in X$ such that $x=T(x, y), y=$ $T(y, x)$.

Proof By the fact that

$$
\frac{p(x, u)+p(y, v)}{2} \leq \max \{p(x, u), p(y, v)\}
$$


for all $x, y, u, v \in X$, the condition (24) implies the condition (7). Therefore, the result as desired follows from Theorem 3.1 and Theorem 3.2.

The following corollary states that $T$ has a fixed point under a certain condition.

Corollary 3.4 In addition to the hypotheses of Corollary 3.3, if $x_{0}$ and $y_{0}$ are comparable, then $T$ has a unique fixed point, that is, there exists $x \in X$ such that $T(x, x)=x$.

Proof Since $x_{0}, y_{0}$ are comparable, we have $x_{0} \geq y_{0}$ or $x_{0} \leq y_{0}$. Suppose we are in the first case. Then, by the mixed monotone property of $T$, we have

$$
x_{1}=T\left(x_{0}, y_{0}\right) \geq T\left(y_{0}, y_{0}\right) \geq T\left(y_{0}, x_{0}\right)=y_{1},
$$

and, hence, by induction one obtains

$$
x_{n} \geq y_{n} \text { for all } n \geq 0
$$

Now, since $x=\lim _{n \rightarrow \infty} x_{n+1}, y=\lim _{n \rightarrow \infty} y_{n+1}$, we have $p(x, y)=\lim _{n \rightarrow \infty} p\left(x_{n+1}, y_{n+1}\right)$. On the other hand, we have

$$
\begin{aligned}
\tau+F\left(p\left(x_{n+1}, y_{n+1}\right)\right) & =\tau+F\left(p\left(T\left(x_{n}, y_{n}\right), T\left(y_{n}, x_{n}\right)\right)\right) \\
& \leq F\left(\max \left\{\left(p\left(x_{n}, x_{n}\right)\right), p\left(y_{n}, y_{n}\right)\right\}\right)
\end{aligned}
$$

Following Lemma 2.7, we also have

$$
\lim _{n \rightarrow \infty} p\left(x_{n}, x_{n}\right)=\lim _{n \rightarrow \infty} p\left(y_{n}, y_{n}\right)=0
$$

Letting $n \rightarrow \infty$ in (25), we arrive at $\lim _{n \rightarrow \infty} p\left(x_{n+1}, y_{n+1}\right)=0$. Therefore $p(x, y)=0$, or $x=y$. Hence $T(x, x)=x$.

Remark 3.5 We underline the fact that the coupled fixed point theorem in this paper can be observed from the fixed point result of a single mapping by using the techniques in [24-26]. On the other hand, we prefer to keep the proofs for the sake of completeness.

\section{Main result: non-cooperative equilibrium problem for two players}

In this section, by using coupled fixed point theorems, we shall show that a two person game has a non-cooperative equilibrium. The reader may consult the excellent sources on general concepts of two person games in [1] and [33].

Let $(S, p)$ be a 0 -complete partial metric space. Suppose that $S$ has a partially order relation $\leq$. We consider a two person game $\mathcal{G}$ in normal form that consists of the following data:

(1) $S_{1}=S$ and $S_{2}=S$ are strategies for player 1 and, respectively, player 2;

(2) the set $U=S_{1} \times S_{2}$ of allowed strategies pairs;

(3) we have the biloss operator

$$
\begin{aligned}
& L: U \rightarrow \mathbb{R}^{2} \\
& \quad\left(s_{1}, s_{2}\right) \mapsto\left(L_{1}\left(s_{1}, s_{2}\right) ; L_{2}\left(s_{1}, s_{2}\right)\right),
\end{aligned}
$$


where $L_{i}\left(s_{1}, s_{2}\right)$ is the loss of player $i$ if the strategies $s_{1}$ and $s_{2}$ are played. A pair $\left(\bar{s}_{1}, \bar{s}_{2}\right) \in U$ is called a non-cooperative equilibrium if

$$
\begin{aligned}
& L_{1}\left(\bar{s}_{1}, \bar{s}_{2}\right) \leq L_{1}\left(s_{1}, \bar{s}_{2}\right), \quad \forall s_{1} \in S_{1}, \\
& L_{2}\left(\bar{s}_{1}, \bar{s}_{2}\right) \leq L_{2}\left(\bar{s}_{1}, s_{2}\right), \quad \forall s_{2} \in S_{2} .
\end{aligned}
$$

This means that

$$
\begin{aligned}
& L_{1}\left(\bar{s}_{1}, \bar{s}_{2}\right)=\min _{s_{1} \in S_{1}} L_{1}\left(s_{1}, \bar{s}_{2}\right), \\
& L_{2}\left(\bar{s}_{1}, \bar{s}_{2}\right)=\min _{s_{2} \in S_{2}} L_{2}\left(\bar{s}_{1}, s_{2}\right) .
\end{aligned}
$$

To see what strategy pairs are non-cooperative equilibria, one considers the optimal decision rules $C, D$ such that

$$
\begin{aligned}
& L_{1}\left(C\left(s_{2}\right), s_{2}\right)=\min _{s_{1} \in S_{1}} L_{1}\left(s_{1}, s_{2}\right), \\
& L_{2}\left(s_{1}, D\left(s_{1}\right)\right)=\min _{s_{2} \in S_{2}} L_{2}\left(s_{1}, s_{2}\right) .
\end{aligned}
$$

Then any fixed point of the map

$$
\left(s_{1}, s_{2}\right) \mapsto\left(C\left(s_{2}\right), D\left(s_{1}\right)\right)
$$

is a non-cooperative equilibrium.

In this section, we shall consider that $D(s)=C(s)$ for all $s \in S$. It is easy to see that if $L_{1}\left(s_{1}, s_{2}\right)=L_{2}\left(s_{2}, s_{1}\right)$ for all $\left(s_{1}, s_{2}\right) \in S_{1} \times S_{2}$ then $D(s)=C(s)$ and it is not difficult to give an example that $D(s)=C(s)$ in the case $L_{1}\left(s_{1}, s_{2}\right) \neq L_{2}\left(s_{2}, s_{1}\right)$. Let $T: S_{1} \times S_{2} \rightarrow \mathbb{R}$ be the map defined by

$$
T(x, y)=C(y)
$$

for all $x, y \in S$. Suppose that $T$ has coupled fixed point $(a, b) \in \mathbb{R}$. It follows that

$$
\begin{aligned}
& a=T(a, b)=C(b), \\
& b=T(b, a)=C(a),
\end{aligned}
$$

and $(a, b)$ is fixed point of the map $\left(s_{1}, s_{2}\right) \mapsto\left(C\left(s_{2}\right), C\left(s_{1}\right)\right)$. Therefore, the existence of the coupled fixed point of $T$ implies a non-cooperative equilibrium. Hence, we can reduce the process of proving the existence of a non-cooperative equilibrium to giving the existence of a coupled fixed point of $T$.

Theorem 4.1 Let $S$ and $\mathcal{G}$ be as mentioned above. Suppose that the optimal decision rule is a monotone continuous function $C$ which satisfies:

(1)

$$
\tau+F(p(C(x), C(y))) \leq F(p(x, y))
$$

for all $x, y \in S$ and $y \geq x$, for some $F \in \mathcal{F}$ and $\tau>0$. 
(2) There are $x_{0}, y_{0} \in \mathbb{R}_{0}^{+}$such that $x_{0} \leq C\left(y_{0}\right), y_{0} \geq C\left(x_{0}\right)$.

Then the two person game $\mathcal{G}$ has a non-cooperative equilibrium.

Proof Let $T: S \times S \rightarrow \mathbb{S}$ be defined by

$$
T(x, y)=C(y)
$$

for all $x, y \in S$. Since $C$ is continuous, we see that $T$ is continuous. Since $C$ is monotone, it is easy to check that $T$ has the mixed monotone property on $X$. For all $x, y, u, v \in \mathbb{R}_{0}^{+}$, with $x \leq u, y \geq v$ we have

$$
p(T(x, y), T(u, v))=p(C(y), C(v)) .
$$

Therefore, the condition (7) reduces to

$$
\tau+F(p(C(y), C(v))) \leq F(\max \{p(x, u), p(y, v)\}),
$$

for every $x \leq u, y \geq v$. Since

$$
\max \{p(x, u), p(y, v)\} \geq p(y, v)
$$

and $F$ is increasing, we see that the condition (31) implies (32). Applying Theorem 3.1, we conclude that $T$ has a coupled fixed point. This implies that the two person game $\mathcal{G}$ has a non-cooperative equilibrium.

Since every metric is partial metric, we immediately obtain the following corollary.

Corollary 4.2 Let $\mathcal{G}$ be as mentioned above. Suppose that $(S, d)$ is a metric space and the optimal decision rule is a monotone continuous function $C$ which satisfies:

(1)

$$
\tau+F(d(C(x), C(y))) \leq F(d(x, y))
$$

for all $x, y \in \mathbb{S}$ and $x<y$, for some $F \in \mathcal{F}$ and $\tau>0$.

(2) There are $x_{0}, y_{0} \in \mathbb{R}_{0}^{+}$such that $x_{0} \leq C\left(y_{0}\right), y_{0} \geq C\left(x_{0}\right)$.

Then the two person game $\mathcal{G}$ has a non-cooperative equilibrium.

Now we shall give an example to show that Corollary 4.2 is effective.

Example 4.3 Consider $S=\mathbb{R}_{0}^{+}$endowed with the metric $d(x, y)=|x-y|$ for all $x, y \in S$. Let $\mathcal{G}$ be a two person game with biloss operator

$$
\begin{aligned}
& L_{1}\left(s_{1}, s_{2}\right)=s_{1}^{2}\left(1+s_{2}\right) e^{-\tau}-2 s_{1}, \\
& L_{2}\left(s_{1}, s_{2}\right)=s_{2}^{2}\left(1+s_{1}\right) e^{-\tau}-2 s_{2},
\end{aligned}
$$

where $s_{1}, s_{2} \in \mathbb{R}_{0}^{+}$and a given $\tau>0$. It is easy to compute the optimal decision rules $C, D$ such that for $\mathcal{G}$

$$
C\left(s_{2}\right)=\frac{e^{-\tau}}{1+s_{2}}
$$


and

$$
D\left(s_{1}\right)=\frac{e^{-\tau}}{1+s_{1}},
$$

where $s_{1}, s_{2} \in \mathbb{R}_{0}^{+}$. We have $D(s)=C(s)$ for all $s \in \mathbb{R}_{0}^{+}$, and $C$ is continuous map. We need show that $C$ satisfies all conditions of Corollary 4.2. We have

$$
d(C(x), C(y))=e^{-\tau}\left|\frac{1}{1+x}-\frac{1}{1+y}\right| \leq e^{-\tau}|x-y|=e^{-\tau} d(x, y)
$$

for all $x, y \in \mathbb{R}_{0}^{+}$. By passing to logarithms, we arrive at

$$
\tau+\ln d(C(x), C(y)) \leq \ln d(x, y)
$$

for all $x \neq y$. Since $F(x)=\ln x \in \mathcal{F}$ we can deduce that $C$ satisfies (1) in Corollary 4.2. Choosing $x_{0}=0$, we have

$$
C\left(x_{0}\right)=e^{-\tau}
$$

Let $y_{0}=1$, we have $y_{0} \geq C\left(x_{0}\right)$. On the other hand $x_{0}=0 \leq C\left(y_{0}\right)=\frac{e^{-\tau}}{2}$. Therefore, $C$ satisfies all conditions of Corollary 4.2. Applying this corollary, we see that the two person game $\mathcal{G}$ has a non-cooperative equilibrium.

\section{Application to nonlinear integral equations}

In this section, we study the existence of unique solution of nonlinear integral equations, as an application of the fixed point theorem proved in Section 3.

Let us consider the following integral equation:

$$
x(t)=h(t)+\int_{0}^{t}\left[K_{1}(t, s)+K_{2}(t, s)\right](f(s, x(s))+g(s, x(s))) d s,
$$

where the unknown function $x(t)$ takes real values.

Let $X=C([0, K])$ be the space of all real continuous functions defined on $[0, K]$. It well known that $C([0, K])$ endowed with the metric

$$
d(x, y)=\|x-y\|=\max _{t \in[0, K]}|x(t)-y(t)|
$$

is a complete metric space. By a solution of (34), we mean a continuous function $x \in X$ that satisfies (34) on $[0, K]$. By certain conditions on $K_{1}, K_{2}, f, g$, and using the results of the previous section, we will prove that (34) has a unique solution. For this, note that $X$ can be equipped with the partial order $\preceq$ given by

$$
\begin{aligned}
x, y \in X, & x \leq y \\
\Longleftrightarrow & (x(t) \leq y(t) \forall t \in[0, K] \text { and }\|x\|,\|y\| \leq 1) \text { or } x(t)=y(t) \forall t \in[0, K] .
\end{aligned}
$$

As in [34] and the references given therein, we assume that the functions $K_{1}, K_{2}, f, g$ fulfill the following conditions. 


\section{Assumption 5.1}

(A) $f, g \in C([0, K] \times \mathbb{R}), h \in X$, and $K_{1}, K_{2} \in C([0, K] \times[0, K])$ such that $K_{1}(t, s) \geq 0$ and $K_{2}(t, s) \leq 0$ for all $t, s \geq 0$;

(B) $f(t, \cdot): \mathbb{R} \rightarrow \mathbb{R}$ is increasing for all $t \in[0, K] ; g(t, \cdot): \mathbb{R} \rightarrow \mathbb{R}$ is decreasing for all $t \in[0, K]$

(C) there exists $\tau \in[1, \infty)$ such that

$$
0 \leq f(t, x)-f(t, y) \leq \tau e^{-\tau} \frac{x-y}{2}, \quad \forall x \geq y
$$

and

$$
-\tau e^{-\tau} \frac{x-y}{2} \leq g(t, x)-g(t, y) \leq 0, \quad \forall x \geq y
$$

(D) $\max _{t, s \in[0, K]}\left|K_{1}(t, s)-K_{2}(t, s)\right| \leq 1$.

Define $T: X \times X \rightarrow X$ by

$$
\begin{aligned}
T(x, y)(t)= & h(t)+\int_{0}^{t} K_{1}(t, s)(f(s, x(s))+g(s, y(s))) d s \\
& +\int_{0}^{t} K_{2}(t, s)(f(s, y(s))+g(s, x(s))) d s
\end{aligned}
$$

for all $t \in[0, K]$.

Definition 5.2 An element $(\alpha, \beta) \in C([0, K] \times C[0, K])$ is a coupled normal lower and a normal upper solution of the integral equation (34) if $\alpha \preceq \beta$ and

$$
\alpha \preceq T(\alpha, \beta) \text { and } \beta \succeq T(\beta, \alpha) .
$$

Theorem 5.3 Suppose that Assumption 5.1 is fulfilled. Then the existence of a coupled normal lower and normal upper solution for (34) provides the existence of a unique solution of $(34)$ in $C([0, K])$.

Proof Suppose $\left\{u_{n}\right\}$ is a monotone non-decreasing sequence in $X$ that converges to $u \in X$. Then, for every $t \in[0, K]$, the sequence of real numbers $u_{1}(t) \leq u_{2}(t) \leq \cdots \leq u_{n}(t) \leq \cdots$ converges to $u(t)$. Moreover, since the normed map is continuous, we can deduce that $\|u\| \leq 1$ provided $\left\|u_{n}\right\| \leq 1$ for all $n$. Therefore, for every $t \in[0, K], n \in \mathbb{N}, u_{n}(t) \leq u(t)$. Hence $u_{n} \leq u$, for all $n \in \mathbb{N}$.

Similarly, we can verify that the limit $v(t)$ of a monotone non-increasing sequence $v_{n}(t)$ in $X$ is a lower bound for all elements in the sequence. That is, $v \leq v_{n}$ for all $n$. Hence, the condition (b) in Corollary 3.3 holds.

For $x \in X$, we defined $\|x\|_{\tau}=\max _{t \in[0, K]}|x(t)| e^{-\tau t}$, where $\tau \geq 1$ is chosen arbitrarily. It is easy to check that $\|\cdot\|_{\tau}$ is a norm equivalent to the maximum norm in $X$ and $X$ endowed with the metric $d_{\tau}$ defined by

$$
d_{\tau}(x, y)=\|x-y\|_{\tau}=\max _{t \in[0, K]}\left\{|x(t)-y(t)| e^{-\tau t}\right\}
$$

for all $x, y \in X$ is a complete metric space. 
Now, consider $X$ endowed with the partial metric given by

$$
p_{\tau}(x, y)= \begin{cases}d_{\tau}(x, y) & \text { if }\|x\|_{\tau} \leq 1,\|y\|_{\tau} \leq 1 \\ d_{\tau}(x, y)+\tau & \text { otherwise }\end{cases}
$$

It is easy to see that $\left(X, p_{\tau}\right)$ is a 0 -complete partial metric space but is not complete (see [27]). We recall that $T: X \times X \rightarrow X$ by

$$
\begin{aligned}
T(x, y)(t)= & h(t)+\int_{0}^{t} K_{1}(t, s)(f(s, x(s))+g(s, y(s))) d s \\
& +\int_{0}^{t} K_{2}(t, s)(f(s, y(s))+g(s, x(s))) d s
\end{aligned}
$$

for all $t \in[0, K]$.

Next, we show that $T$ has the mixed monotone property. Indeed, for $x_{1}, x_{2} \in C([0, K])$ and $x_{1} \leq x_{2}$, that is, $x_{1}(t) \leq x_{2}(t)$, for every $t \in[0, K]$, we have

$$
\begin{aligned}
T\left(x_{1}, y\right)(t)-T\left(x_{2}, y\right)(t)= & \int_{0}^{t} K_{1}(t, s)\left[f\left(s, x_{1}(s)\right)+g(s, y(s))\right] d s \\
& +\int_{0}^{t} K_{2}(t, s)\left[f(s, y(s))+g\left(s, x_{1}(s)\right)\right] d s+h(t) \\
& -\int_{0}^{t} K_{1}(t, s)\left[f\left(s, x_{2}(s)\right)+g(s, y(s))\right] d s \\
& -\int_{0}^{t} K_{2}(t, s)\left[f(s, y(s))+g\left(s, x_{2}(s)\right)\right] d s-h(t) \\
= & \int_{0}^{t} K_{1}(t, s)\left[f\left(s, x_{1}(s)\right)-f\left(s, x_{2}(s)\right)\right] d s \\
& +\int_{0}^{t} K_{2}(t, s)\left[g\left(s, x_{1}(s)\right)-g\left(s, x_{2}(s)\right)\right] d s \leq 0,
\end{aligned}
$$

for every $t \in[0, K]$, by Assumption 5.1. This yields $T\left(x_{1}, y\right)(t) \leq T\left(x_{2}, y\right)(t)$, for every $t \in$ $[0, K]$, that is, $T\left(x_{1}, y\right) \leq T\left(x_{2}, y\right)$. By the same computation, we arrive at $T\left(x, y_{1}\right) \leq T\left(x, y_{2}\right)$ if $y_{1} \geq y_{2}$. Hence, $T$ has the mixed monotone property.

Now, for $x \geq u$ and $y \leq v$, we have

$$
\begin{aligned}
|T(x, y)(t)-T(u, v)(t)| & \mid\left[\int_{0}^{t} K_{1}(t, s)(f(s, x(s))+g(s, y(s))) d s\right. \\
& \left.+\int_{0}^{t} K_{2}(t, s)(f(s, y(s))+g(s, x(s))) d s+h(t)\right] \\
& -\left[\int_{0}^{t} K_{1}(t, s)(f(s, u(s))+g(s, v(s))) d s\right. \\
& \left.+\int_{0}^{t} K_{2}(t, s)(f(s, v(s))+g(s, u(s))) d s+h(t)\right] \mid \\
= & \mid \int_{0}^{t} K_{1}(t, s)[(f(s, x(s))-f(s, u(s)))+(g(s, y(s))-g(s, v(s)))] d s
\end{aligned}
$$




$$
\begin{aligned}
& +\int_{0}^{t} K_{2}(t, s)[(f(s, y(s))-f(s, v(s)))+(g(s, x(s))-g(s, u(s)))] d s \mid \\
= & \mid \int_{0}^{t} K_{1}(t, s)[(f(s, x(s))-f(s, u(s)))-(g(s, v(s))-g(s, y(s)))] d s \\
& -\int_{0}^{t} K_{2}(t, s)[(f(s, v(s))-f(s, y(s)))-(g(s, x(s))-g(s, u(s)))] d s \mid \\
\leq & \mid \int_{0}^{t} K_{1}(t, s) \tau e^{-\tau}\left[\frac{x(s)-u(s)}{2}+\frac{v(s)-y(s)}{2}\right] d s \\
& -\int_{0}^{t} K_{2}(t, s) \tau e^{-\tau}\left[\frac{v(s)-y(s)}{2}+\frac{x(s)-u(s)}{2}\right] d s \mid \\
\leq & \tau e^{-\tau} \int_{0}^{t}\left|\left[K_{1}(t, s)-K_{2}(t, s)\right]\left[\frac{x(s)-u(s)}{2}+\frac{v(s)-y(s)}{2}\right]\right| d s \\
= & \tau e^{-\tau} \int_{0}^{t}\left|K_{1}(t, s)-K_{2}(t, s)\right| e^{\tau s}\left[\frac{|x(s)-u(s)| e^{-\tau s}}{2}+\frac{|x(s)-u(s)| e^{-\tau s}}{2}\right] d s \\
\leq & \tau e^{-\tau} \int_{0}^{t} \max _{t, s \in[0, K]}\left|K_{1}(t, s)-K_{2}(t, s)\right| e^{\tau s}\left[\frac{\|x-u\|_{\tau}}{2}+\frac{\|y-v\|_{\tau}}{2}\right] d s \\
\leq & \tau e^{-\tau} \frac{e^{\tau t}}{\tau}\left[\frac{\|x-u\|_{\tau}}{2}+\frac{\|y-v\|_{\tau}}{2}\right] .
\end{aligned}
$$

It follows that

$$
|T(x, y)(t)-T(u, v)(t)| e^{-\tau t} \leq e^{-\tau}\left[\frac{\|x-u\|_{\tau}}{2}+\frac{\|y-v\|_{\tau}}{2}\right] .
$$

Hence, for all $x, y, u, v \in X$ such that $x \geq u$ and $y \leq v$, since $\|x\|_{\tau},\|y\|_{\tau},\|u\|_{\tau},\|v\|_{\tau} \leq 1$, we have

$$
p_{\tau}(T(x, y), T(u, v)) \leq e^{-\tau} \frac{1}{2}\left[p_{\tau}(x, u)+p_{\tau}(y, v)\right] .
$$

By passing to logarithms, we arrive at

$$
\tau+\ln p_{\tau}(T(x, y), T(u, v)) \leq \ln \left(\frac{p_{\tau}(x, u)+p_{\tau}(y, v)}{2}\right) .
$$

Since $F(x)=\ln x \in \mathcal{F}$, we conclude that $T$ satisfies the condition (24). Now, let $(\alpha, \beta)$ be a coupled normal lower and normal upper solution of the integral equation of (34). Then we have $\alpha \preceq \beta$,

$$
\alpha \preceq T(\alpha, \beta) \text { and } \beta \succeq T(\beta, \alpha) \text {. }
$$

Finally, applying Corollary 3.4, we can conclude that $T$ has a fixed point $x$. Hence $T(x, x)=$ $x$ and $x$ is an unique solution of (34).

The authors declare that they have no competing interests. 


\section{Author details}

'Department of Mathematics, Vinh University, 182 Le Duan, Vinh City, Vietnam. ${ }^{2}$ Nonlinear Analysis and Applied Mathematics Research Group (NAAM), Department of Mathematics, Faculty of Science, King Abdulaziz University, P.O. Box 80203, Jeddah, 21589, Saudi Arabia. ${ }^{3}$ Department of Mathematics, Atilim University, Incek, Ankara, 06836, Turkey. ${ }^{4}$ Nonlinear Analysis and Applied Mathematics Research Group (NAAM), King Abdulaziz University, Jeddah, 21589, Saudi Arabia.

\section{Acknowledgements}

The authors are grateful to the reviewers for their careful reviews and useful comments. This article was funded by the Deanship of Scientific Research (DSR), King Abdulaziz University. The authors, therefore, acknowledge with thanks DSR technical and financial support.

\section{Received: 3 March 2015 Accepted: 27 April 2015 Published online: 12 May 2015}

\section{References}

1. Christian, OE: Games, Fixed Points and Mathematical Economics. School of Economics and Finance University of St. Andrews. http://ssrn.com/abstract=976592 (2003)

2. Mathews, GS: Partial metric topology, Reseach Report 212. Department of Computer Science University of Warwick (1992)

3. Mathews, GS: Partial metric topology. Ann. N.Y. Acad. Sci. 728, 183-197 (1994)

4. Abdeljawad, T, Karapınar, E, Taş, K: Existence and uniqueness of common fixed point on partial metric spaces. Appl. Math. Lett. 24(11), 1900-1904 (2011)

5. Ilić, D, Pavlović, V, Rakocević, V: Some new extensions of Banach's contraction principle to partial metric space. Appl. Math. Lett. 24(8), 1326-1330 (2011)

6. Gulyaz, S, Karapınar, E: Coupled fixed point result in partially ordered partial metric spaces through implicit function. Hacet. J. Math. Stat. 42, 347-357 (2013)

7. Karapınar, E: Weak $\varphi$-contraction on partial metric spaces. J. Comput. Anal. Appl. 14, 206-210 (2012)

8. Karapınar, E: Generalizations of Caristi Kirk's theorem on partial metric spaces. Fixed Point Theory Appl. (2011) doi:10.1186/1687-1812-2011-4

9. Karapınar, E, Erhan, ÝM, Ulus, AY: Fixed point theorem for cyclic maps on partial metric spaces. Appl. Math. Inf. Sci. 6 , 239-244 (2012)

10. Bukatin, M, Kopperman, R, Matthews, S, Pajoohesh, H: Partial metric spaces. Am. Math. Mon. 116(8), 708-718 (2009)

11. Chi, KP, Karapınar, E, Thanh, TD: A generalized contraction principle in partial metric spaces. Math. Comput. Model. 55(5-6), 1673-1681 (2012)

12. Chi, KP, Karapınar, E, Thanh, TD: On the fixed point theorems for generalized weakly contractive mappings on partial metric spaces. Bull. Iran. Math. Soc. 39(2), 369-381 (2013)

13. Oltra, S, Valero, O: Banach's fixed point theorem for partial metric spaces. Rend. Ist. Mat. Univ. Trieste 36(1-2), 17-26 (2004)

14. Valero, O: On Banach fixed point theorems for partial metric spaces. Appl. Gen. Topol. 6(2), 229-240 (2005)

15. Romaguera, S: A Kirk type characterization of completeness for partial metric spaces. Fixed Point Theory Appl. 2010 Article ID 493298 (2010)

16. Ran, ACM, Reurings, MCB: A fixed point theorem in partially ordered sets and some applications to matrix equations. Proc. Am. Math. Soc. 132, 1435-1443 (2004)

17. Nieto, J, Rodriguez-Lopez, R: Contractive mapping theorems in partially ordered sets and applications to ordinary differential equations. Order 22, 223-239 (2005)

18. Nieto, J, Rodriguez-Lopez, R: Existence and uniqueness of fixed point in partially ordered sets and applications to ordinary differential equations. Acta Math. Sin. 23, 2205-2212 (2007)

19. Guo, D, Lakshmikantham, V: Coupled fixed points of nonlinear operators with applications. Nonlinear Anal. TMA 11, 623-632 (1987)

20. Gnana Bhaskar, T, Lakshmikantham, V: Fixed point theorems in partially ordered metric spaces and application. Nonlinear Anal. 65, 1379-1393 (2006)

21. Lakshmikantham, V, Ćirić, L: Coupled fixed point theorems for nonlinear contractions in partially ordered metric spaces. Nonlinear Anal. 70, 4341-4349 (2009)

22. Agarwal, RP, El-Gebeily, MA, O'Regan, D: Generalized contractions in partially ordered metric spaces. Appl. Anal. 87, 1-8 (2008)

23. Luong, NV, Thuan, NX: Coupled point theorems in partially ordered metric spaces and applications. Nonlinear Anal. 74, 983-992 (2011)

24. Samet, B, Karapınar, E, Aydi, H, Rajic, V: Discussion on some coupled fixed point theorems. Fixed Point Theory Appl. 2013, Article ID 50 (2013)

25. Rad, GS, Shukla, S, Rahimi, H: Some relations between $n$-tuple fixed point and fixed point results. Rev. R. Acad. Cienc Exactas Fís. Nat., Ser. A Mat. (2014). doi:10.1007/s13398-014-0196-0

26. Roldan, A, Martinez-Moreno, J, Roldan, C, Karapınar, E: Some remarks on multidimensional fixed point theorems. Fixed Point Theory 15(2), 545-558 (2014)

27. Paesano, D, Vetro, C: Multi-valued F-contractions in 0-complete partial metric spaces with application to Volterra type integral equation. Rev. R. Acad. Cienc. Exactas Fís. Nat., Ser. A Mat. 108(2), 1005-1020 (2014)

28. Wardowski, D: Fixed points of a new type of contractive mappings in complete metric spaces. Fixed Point Theory Appl. (2012). doi:10.1186/1687-1812-2012-94

29. Alsulami, HH, Karapınar, E, Piri, H: Fixed points of generalized F-Suzuki type contraction in complete metric-like spaces. Discrete Dyn. Nat. Soc. 2015, Article ID 969726 (2015)

30. Alsulami, HH, Karapınar, E, Piri, H: Fixed points of modified F-contractive mappings in complete metric-like spaces. J. Funct. Spaces 2014, Article ID 270971 (2014)

31. Sgroi, M, Vetro, C: Multi-valued F-contractions and the solution of certain functional and integral equations. Filomat $27,1259-1268(2013)$ 
32. Cosentino, M, Vetro, P: Fixed point results for F-contractive mappings of Hardy-Rogers-type. Filomat 28, 715-722 (2014)

33. Border, KC: Fixed Point Theorems with Applications to Economics and Game Theory. Cambridge University Press, Cambridge (1989)

34. Berinde, V: Generalized coupled fixed point theorems for mixed monotone mappings in partially ordered metric spaces. Nonlinear Anal. 74, 7347-7355 (2011)

Submit your manuscript to a SpringerOpen ${ }^{\circ}$ journal and benefit from:

- Convenient online submission

- Rigorous peer review

- Immediate publication on acceptance

- Open access: articles freely available online

- High visibility within the field

Retaining the copyright to your article

Submit your next manuscript at $\gg$ springeropen.com 